\title{
Article \\ Preliminary Propulsion and Power System Design of a Tandem-Wing Long-Range eVTOL Aircraft
}

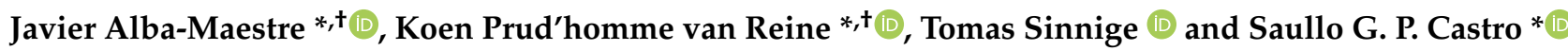 \\ Faculty of Aerospace Engineering, Delft University of Technology, Kluyverweg 1, 2629 HS Delft, \\ The Netherlands; T.Sinnige@tudelft.nl \\ * Correspondence: J.AlbaMaestre@student.tudelft.nl (J.A.-M.); \\ K.PrudhommevanReine@student.tudelft.nl (K.P.v.R.); S.G.P.Castro@tudelft.nl (S.G.P.C.) \\ † These authors contributed equally to this work.
}

Citation: Alba-Maestre, J.;

Prud'homme van Reine, K.; Sinnige,

T.; Castro, S.G.P. Preliminary

Propulsion and Power System Design of a Tandem-Wing Long-Range eVTOL Aircraft. Appl. Sci. 2021, 11, 11083. https://doi.org/10.3390/ app112311083

Academic Editor: Ho Yon Hwang

Received: 20 October 2021

Accepted: 18 November 2021

Published: 23 November 2021

Publisher's Note: MDPI stays neutral with regard to jurisdictional claims in published maps and institutional affiliations.

Copyright: (c) 2021 by the authors. Licensee MDPI, Basel, Switzerland. This article is an open access article distributed under the terms and conditions of the Creative Commons Attribution (CC BY) license (https:/ / creativecommons.org/licenses/by/ $4.0 /)$.

\begin{abstract}
Novel eVTOL aircraft configurations are picking up momentum in the emerging market of urban air mobility (UAM). These configurations feature electrical power systems and distributed propulsion architectures, both uncommon in current aircraft. As such, the design of eVTOL aircraft lies outside the bounds of current established frameworks and poses many challenges in the field of preliminary aircraft design. This paper presents a preliminary design methodology for open rotor eVTOL configurations with batteries as the power source. First, the propeller external dimensions are calculated, and then an optimised blade geometry for cruise condition is computed. Thereupon, the batteries and electric motors are sized. The design framework is then applied to an eVTOL aircraft with a design range of $400 \mathrm{~km}$ and a capacity of five occupants (four passengers and one pilot), focusing on the central-European market and aimed to be released in 2030. The final configuration is a battery-powered tandem-wing aircraft with 12 variable-pitch, variable-speed open rotors placed on the leading edges of the wings. These rotors rotate outboard-down and feature six blades. The power source comprises 24 solid-state lithium batteries with a nominal voltage of $500 \mathrm{~V}$ and an assumed energy density of $500 \mathrm{Wh} / \mathrm{kg}$. The proposed design methodology offers the possibility of computing the necessary propeller geometry for numerical simulations in the early stages of the design, and of easily obtaining accurate estimates for the mass of the power system which can improve the overall mass estimates for the analysed configuration.
\end{abstract}

Keywords: eVTOL; distributed electric propulsion; battery; preliminary design; urban air mobility; tandem wing

\section{Introduction}

The widespread interest in electric vertical take-off and landing (eVTOL) vehicles has accrued ample momentum in recent years to spark a new flying revolution. However, several technological and socioeconomic obstacles lie before the widespread adoption of urban air mobility (UAM) vehicles such as eVTOLs. In order to bridge some of these gaps, a multidisciplinary design of one such concept was conducted as part of a final B.Sc. project [1-3] at the Delft University of Technology. This project features the study and preliminary design of a long-range eVTOL aircraft through five different disciplines: aerodynamics, propulsion and power, stability and control, structures, and flight performance; and includes the preliminary multidisciplinary design optimisation of the configuration.

\subsection{Proposed Configuration}

The aircraft designed in the project, designated as Wigeon, has a tandem-wing configuration and leading edge open rotors, a capacity of four passengers and one pilot, a range of $400 \mathrm{~km}$, and is fully electric. The transition between horizontal and vertical flight is achieved via the rotation of the wings. The final configuration can be seen in Figure 1. 
The proposed characteristics stem from a market analysis conducted during the project, targeting market entry in 2030 [3]. To achieve this target date, technology-readiness levels expected in 2030 were used in the design process, and certification was taken into account by following the Special Condition for small-category VTOL aircraft by EASA [4], all of which is further detailed in the original project report [3]. The project primarily focuses on the central-European market, where this type of configuration could achieve an $8 \%$ market share by 2035, and is also expected to reach a market share of $2 \%$ in the North America and Asia-Pacific regions [3]. The market analysis concluded that a $400 \mathrm{~km}$ range opens the possibility of connecting big European cities, such as Paris and London, also offering wide-ranging connections between other main European cities and facilitating connections between mainland Europe and the islands surrounding it. Furthermore, the Wigeon has been designed with the objective of easy market introduction, without requiring an extensive infrastructure for its operation, nor extensive certification campaigns for critical systems. As such, the selected power source was that of batteries instead of hydrogen [2] and its size is limited to be operable in existing helipads, for which all horizontal dimensions are constrained to be below $14 \mathrm{~m} \mathrm{[3].}$

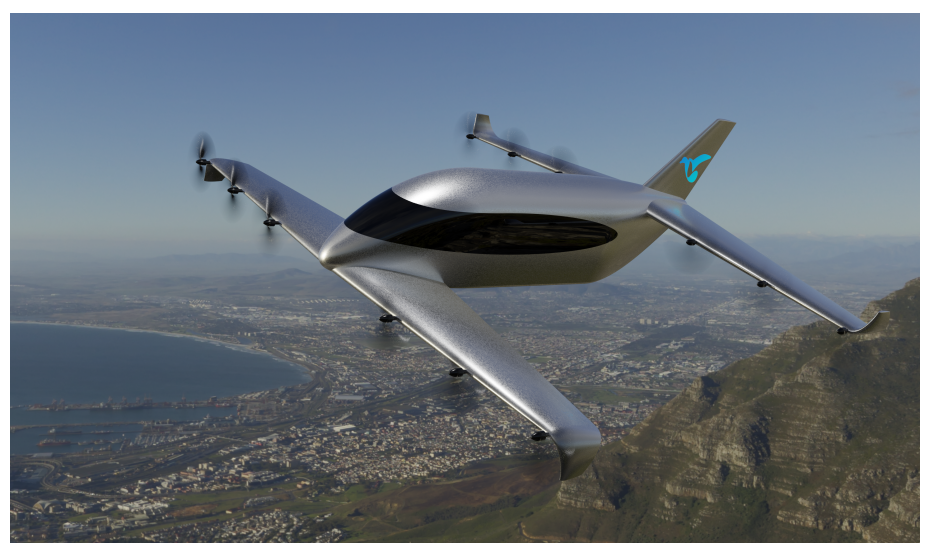

Figure 1. Rendering of the final design of the analysed configuration of the Wigeon.

\subsection{Background}

This manuscript elaborates on the propulsion and power system design methodology devised in the Wigeon project as a means of the preliminary design of open rotor eVTOL configurations, which are characterised by the use of innovative distributed propulsion architectures for their propulsion and power systems. Procedures to design efficient propellers are already available in the literature [5,6] and can be applied to eVTOL aircraft; however, the use of these unconventional distributed propulsion configurations, required in order to ensure safety, controllability, and performance during vertical and horizontal flight and the transition between them also results in more difficult performance analysis due to the close coupling between the propulsive and aerodynamic systems, even though analyses of these open rotor distributed propulsion configurations applied to VTOL $[7,8]$ and UAV [9] vehicles are already available in the literature.

The wide range of operating conditions of eVTOL aircraft also presents a challenge for the choice of an optimal design which encompasses all of the mission conditions. Moreover, the power system of eVTOL aircraft usually features fully electric energy sources, which are not commonly used in commercial aircraft and provide a technological challenge due to their lower current performance when compared to conventional combustion aircraft. This study aimed to tackle these challenges with a simple design methodology for the early performance estimation of such systems, by applying the available methods and studies into a combined design framework for the propulsion and power systems of open rotor battery-powered eVTOL aircraft. 


\subsection{Overview of the Design Procedure}

The design procedure presented in this paper is summarised in Figure 2. The design procedure starts from a set of inputs, which are the mission profile, the preliminary external dimensions of the vehicle, and a preliminary estimation of the flight performance characteristics of the aircraft (required energy and power for the mission), all of which come from the original project [3]. The propulsion and power design procedures run in parallel until a satisfactory final design is reached for both of them.

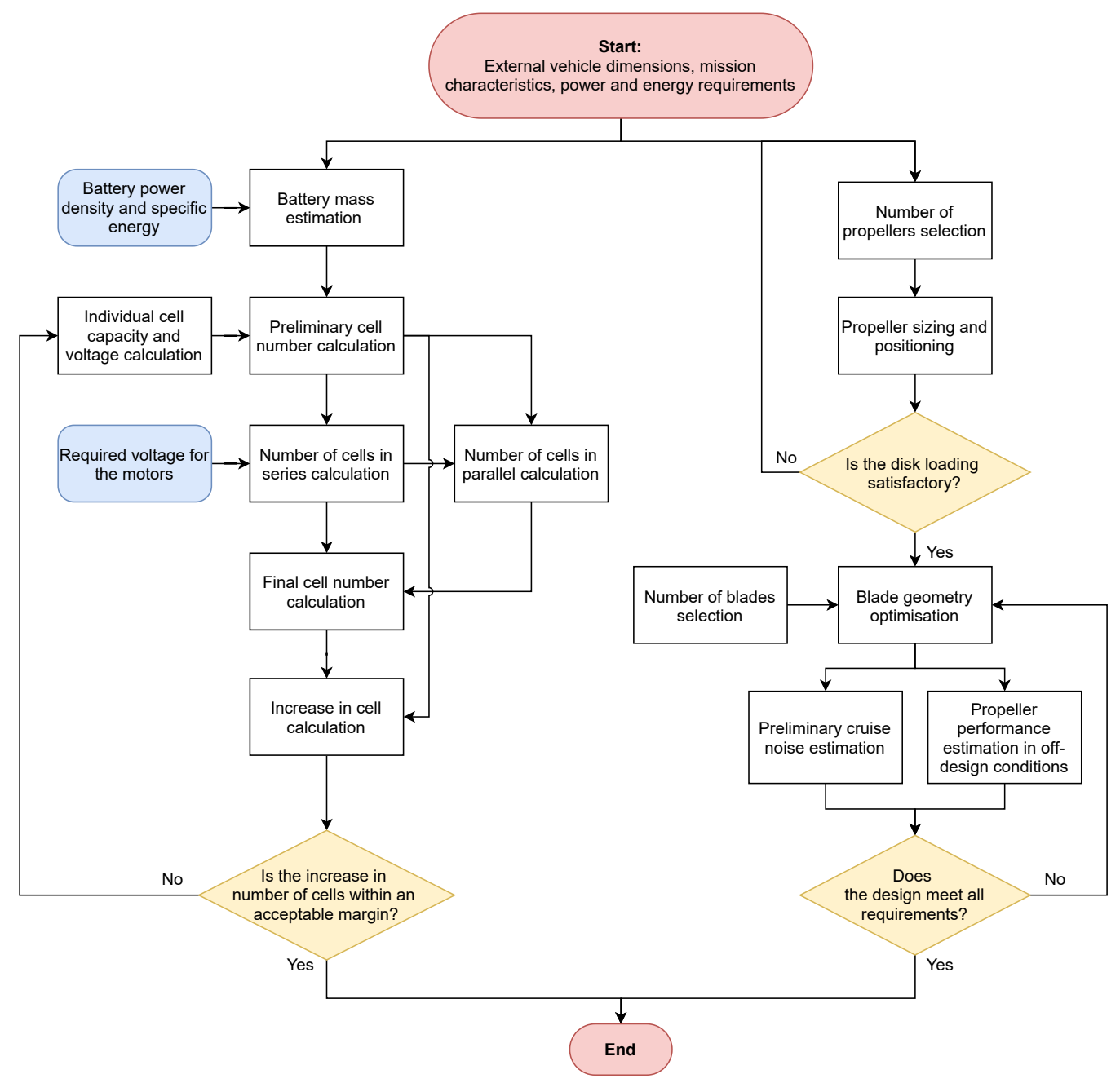

Figure 2. Flowchart of the propulsion and power systems design procedure presented in this paper.

\section{Propulsion System Design Methodology}

The goal of the propulsion design framework is to allow the preliminary study and comparison of different configurations. The analysis focuses around the design of efficient propellers which can provide enough thrust during all phases of the mission, which are [3]: vertical take-off (1); transition from hover to climb (2); climb to cruising altitude (3); cruise (4); loiter in horizontal flight (5); descent (6); transition to hover (7); loiter in hover (8); and landing (9). A schematic of these phases can be seen in Figure 3. 


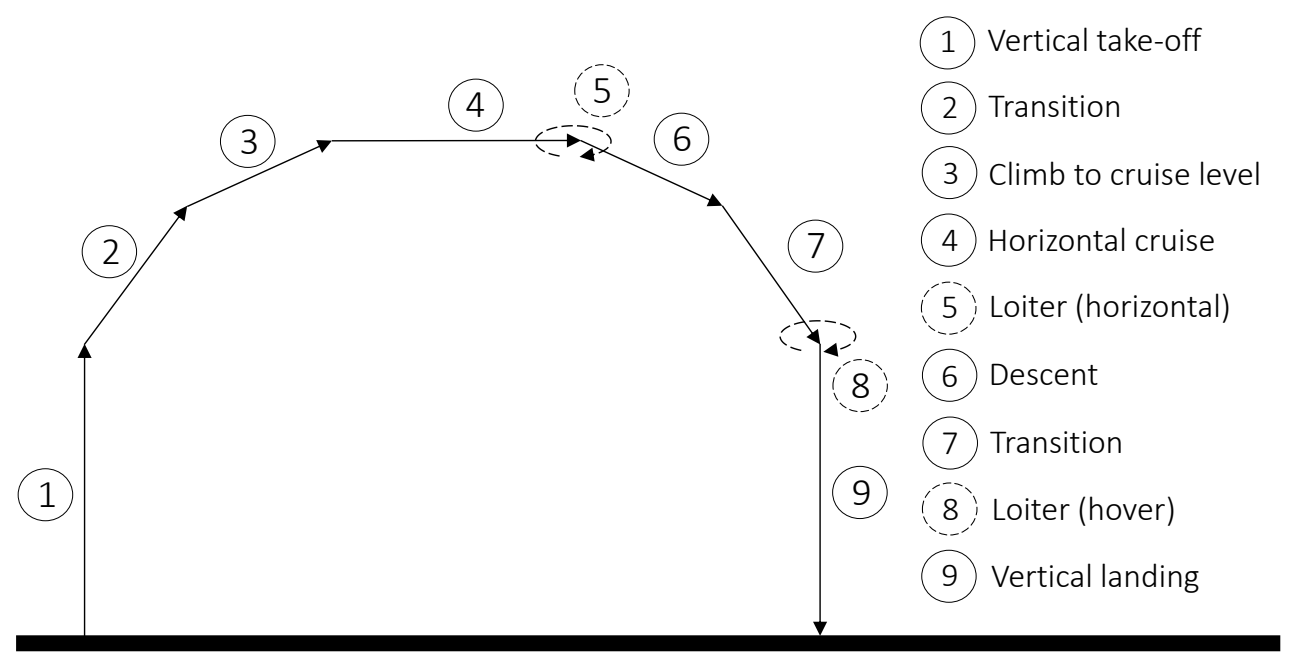

Figure 3. Schematic of the 9 flight phases of a regular eVTOL flight mission.

\subsection{Design of the Propeller Blades}

The main tool used in this framework for the preliminary design of the propeller blades is the blade element momentum theory (BEMT), a theory which analyses the performance of propeller blades by dividing each individual blade into a number of stations, coupling this to a momentum analysis for each individual annulus of the propeller streamtube. These stations, each with a different twist angle, were then analysed as 2D airfoils in order to compute their individual thrust and drag, and later added to obtain the overall performance of the propeller blade. Correction factors to account for 3D effects are also included in the process to improve the accuracy of the method.

In order to achieve an efficient blade design, the procedure laid down by Adkins and Liebeck [5] was used. This procedure was based on a similar work by Larrabee [6] and modified Larrabee's equations to eliminate small angle approximations and extend the procedure from lightly to moderately and highly loaded propellers.

The procedure yields the blade geometry that minimises the propeller induced and profile losses at a chosen design condition. The condition for which this happens is when the product of the local radius of a blade station times the tangent of the local flow angle at such station remains constant throughout the blade $(r \tan (\Phi)=$ const.) [5], a condition which results in the vortex sheet shed by the propeller having the form of a regular screw surface. This condition was originally described by Albert Betz [10], and is thus referred to as the Betz condition. The original work by Adkins and Liebeck includes further derivations to support the equations used such that only the final equations are herein presented.

From [5], the term $\tan (\Phi)$ can be rewritten as Equation (1), where $\lambda$ is the tip speed ratio, defined as the ratio between the inflow speed and the tangential speed of the propeller tip $(V / \Omega R)$ and $\xi$ is the non-dimensional radius of the propeller, defined as the ratio between the local and the propeller radii $(r / R)$. With this, Equation (1) can be rewritten as Equation (2):

$$
\begin{aligned}
\tan (\Phi) & =\left(1+\frac{\zeta}{2}\right) \frac{\lambda}{\xi} \\
r \tan (\Phi) & =\left(1+\frac{\zeta}{2}\right) R \lambda
\end{aligned}
$$

Since the displacement velocity ratio $(\zeta$, defined as the ratio between the displacement velocity of the vortex sheet and the inflow speed), $\lambda$ and $R$ are constants, this agrees with the aforementioned Betz condition: $r \tan (\Phi)$ is constant. 
To define the chord of each blade station, Equation (3) [5] can be used:

$$
w c=\frac{4 \pi \lambda R \zeta V G}{B C_{l}}
$$

In this equation, $G$ is a parameter defined in Equation (4) where $F$ represents the momentum loss factor, defined as Equation (5) [5]:

$$
\begin{gathered}
G=F \sin (\Phi) \cos (\Phi) \\
F=\frac{2}{\pi} \arccos \left(e^{-\frac{B}{2} \frac{1-\tilde{\xi}}{\sin \left(\Phi_{\text {tip }}\right)}}\right)
\end{gathered}
$$

To define the blade geometry, the chord length and pitch angle of each station are needed. The local pitch angle of the blade, $\beta$, is defined as the angle between the disk plane and the chord of the blade airfoil. As can be seen in Figure 4, it follows that the angle of attack of the section is defined as $\alpha=\beta-\Phi$ :

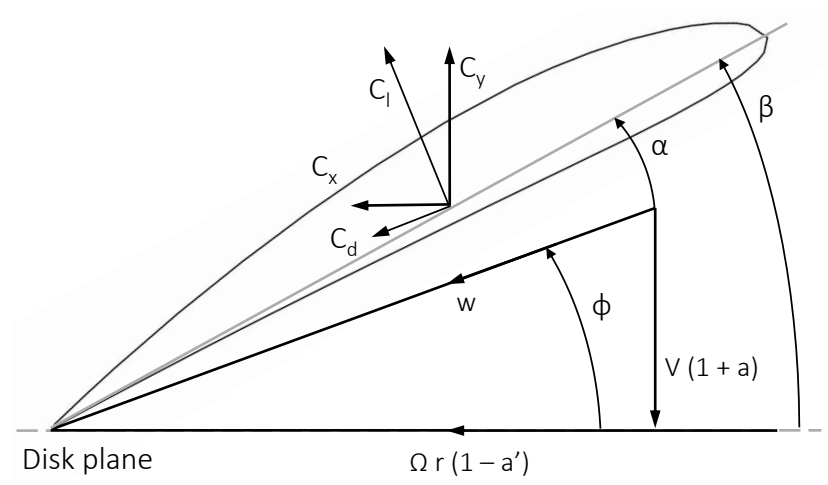

Figure 4. Velocities acting on the blade station with their corresponding angles. Nomenclature following [5].

Equation (2) allows one to calculate the required flow angle at each blade station, where $R$ and $\lambda$ are known. The angle of attack is then chosen such that the $C_{l} / C_{d}$ of the blade is maximised for each station, while maintaining a reasonable value for $C_{l}$ that allows the propeller to generate sufficient thrust. For this, the lift coefficient is first chosen, and the corresponding drag coefficient and angle of attack are then retrieved from known airfoil data. The optimum angle is not the same for every station as it depends on the Reynolds number with respect to the blade chord and local effective velocity, which increases moving outboard on the blade. The procedure used to obtain the airfoil data in this paper is explained later. For the chord, if the product $w c$ is calculated from Equation (3), and from the blade geometry shown in Figure $4, w$ can be defined as $V(1+a) / \sin (\Phi)$. The chord can then be calculated as $c=w c / w$.

In both equations, the displacement velocity ratio $\zeta$ is needed and it needs to be iteratively calculated, as it is not known beforehand. This procedure is not the focus of this paper, but rather a tool used in the design, and it has already been detailed by the original authors [5] and explained in greater detail in the original project report [3]. Thus, the calculation of the displacement velocity ratio is not further detailed in the present paper.

\section{Implementation of the Blade Design Procedure in the Design Framework}

The procedure takes as inputs the working conditions of the propeller at the design point and the predefined characteristics of the propeller geometry, and it optimises the blade shape to maximise the efficiency in the design condition. The working conditions are the flight speed, the air properties at the design point, the thrust that needs to be delivered by the propeller, and the rotational speed that the propeller operates at. The propeller 
geometry that needs to be predefined comprises the number of blades, the propeller radius, the radius of the propeller hub, and the lift and drag coefficients of the airfoil used in the blades.

The propeller radius comes from the method outlined in Section 2.2 and an initial guess for the radius of the propeller hub can be estimated from existing propellers with similar radii and working conditions. The number of blades is a parameter that needs to be optimised for the final design of the aircraft. However, a reasonable initial estimate can be found by taking into account the effects of the number of blades. More blades result in less noise, since the pressure increment per blade is smaller (for the same total thrust), and can also result in higher efficiency. However, having more blades implies that they will have lower chord values for the same span (higher aspect ratio), since, for a fixed set of inputs, modifying only the number of blades results in propellers with the same solidity [6]. If the aspect ratio becomes too high, this can lead to structural or manufacturing issues. Moreover, introducing a pitch control mechanism for more blades can increase its complexity. Finding a balance for these characteristics is necessary for a detailed, optimised design, but a reasonable estimate suffices for the procedure used, as the result is not highly sensible to the chosen number of blades (a sensitivity analysis is presented in Section 3.2.2). The flight speed and air properties at the design point and the thrust needed from the propeller may be obtained from the mission analysis of the desired configuration and a preliminary flight performance estimate.

For this paper, a NACA4412 was selected as the airfoil for the blades [3] with its performance analysed using XFOIL [11] through which the airfoil is simulated at a wide range of Reynolds numbers and angles of attack in order to create a database for the airfoil performance. With this, during the design process, the lift and drag coefficients of the airfoil at a certain angle of attack and Reynolds number can be retrieved. To account for compressibility, the Mach number at each station is calculated from the known local effective flow velocity $w$, and the Prandtl-Glauert correction factor is then applied. It is worth mentioning that the overall performance of the propeller will be hindered by the fact that only one airfoil is used for the entire blade. As mentioned above, outer sections of the blade see higher Reynolds and Mach numbers, which implies that different types of airfoils, and different thickness-to-chord ratios, would offer the best performance at different sections of the blade. Including these considerations in the method, however, would increase the complexity beyond what is desired for this preliminary design framework, and is thus left for further stages of the design.

The outputs of the blade design procedure are the chord length and pitch angle of each blade section. The blade sections are aligned at the quarter-chord (i.e., the quarter-chord sweep is zero), and the lean of the propellers is set to zero. These parameters have been assumed and are not part of the design process. With this, together with the geometry of the airfoil, and the known parameters such as the radius and number of blades, the geometry of the propeller is fully defined.

\subsection{Propeller Positioning and Sizing}

The propeller radius is necessary for the design of the blades, and the first step adopted herein for the size the radius of the propellers is to select the number and positioning of the propellers. Since the nature of the design framework of this paper is preliminary, the design point of the aircraft is to be chosen semi-qualitatively based on a series of important considerations detailed below. The blade geometry of the propellers is to then be optimised for this design point.

The use of distributed electric propulsion (DEP) architectures in eVTOL aircraft greatly widens the design space with respect to conventional architectures, which allows for more optimised designs, but consequently complicates the selection of an initial estimate for the design point. In practice, a reasonable initial estimate can be obtained based on a series of requirements and constraints that stem from the architecture of the aircraft, from the nature 
of the operations that the aircraft is being designed for, and from general considerations on the performance of the aircraft.

\subsubsection{Number of Propellers}

The selection of the number of propellers is based on assessing the benefits of having fewer and bigger propellers versus more and smaller propellers. These choices have an effect on several performance characteristics of the aircraft:

1. Disk loading: Bigger propellers allow for a larger total area and hence a lower disk loading which improve efficiency. This directly translates into a lower required peak power which can decrease the size (and thus weight) of the electric motors and the power system [2];

2. Ground clearance: More propellers means smaller radii, which therefore allows for more ground clearance when the wings are in horizontal position;

3. Propeller-wing interaction: Bigger propellers have a higher slipstream height which results in a higher increase in lift due to the propeller slipstream for the same slipstream velocity [12]. On the other hand, bigger propellers would reduce the slipstream velocity;

4. Propeller-propeller interaction: For the same front and rear wing separation, smaller front propellers with lower slipstream heights allow one to more easily place them such that the propellers on the downstream wing are outside the slipstream of the propellers on the upstream wing. This slipstream ingestion can lead to big losses in thrust from the second row of propellers [7] and increase noise emissions;

5. Blade rotation mechanism: The propellers need a mechanism to alter the pitch of the blades. With very small propellers, implementing such a mechanism becomes more difficult.

6. Safety in OEI conditions: More propellers mean more redundancy, and with more and smaller propellers, a failure of one of them has a smaller effect on the controllability in hover and on total thrust.

The proposed aircraft architecture features two wings. For the present framework, it was decided to place the same number of propellers, all with the same size, on each wing, for two reasons. First, to evenly distribute the thrust and to minimise differences in propeller loading. Here, it is worth noting that due to the fact that the CG of the aircraft is closer to the front wing, the front propellers need to provide higher thrust during hover. However, (due to reason 4) in the previous list, having less but bigger propellers in the front wing than in the back wing is deemed not necessarily beneficial overall; due to the fact that hover is a very small part of the mission compared to cruise, and the potential slipstream ingestion caused by bigger front propellers would lead to thrust reduction during the cruise and hence a need for more power during the entire cruise phase of the mission. Secondly, having all propellers with the same size is simpler and cheaper for manufacturing and maintenance, since only one electrical motor and two different blade designs are needed (due to the fact that the propellers are rotating in opposite directions on each side of the fuselage, as further discussed in Section 2.2.2). In more advanced stages of the design process (not covered in this design framework), where more information about the performance of the aircraft is known, this assumption can be revised, and the relative sizes of the propellers can be adjusted.

Based on the previous considerations, the possible number of propellers considered were 8,12 , and 16 , which, respectively, translates to 2,3 , and 4 per half wing. Having only 1 propeller per half wing was discarded due to the lack of redundancy and having more propellers would mean having very small propellers which would therefore be too highly loaded, leading to low propulsive efficiency.

\subsubsection{Positioning and Sizing}

The second step in the design process is to select the placement of the propellers and assess the available space for the propellers. The outermost propellers of the selected 
eVTOL configuration are wingtip-mounted: first, this allows one to maximise the radius of the propellers and thus the area for a given wingspan, which results in a lower disk loading; secondly, having outboard rotating propellers at the tips of the wings can help counteract the wingtip vortices due to the propeller-induced swirl, hence reducing the induced drag of the wing [13]. Figure 5 shows a schematic diagram of the positioning of the propellers and of the geometrical parameters that define the sizing and positioning procedure.

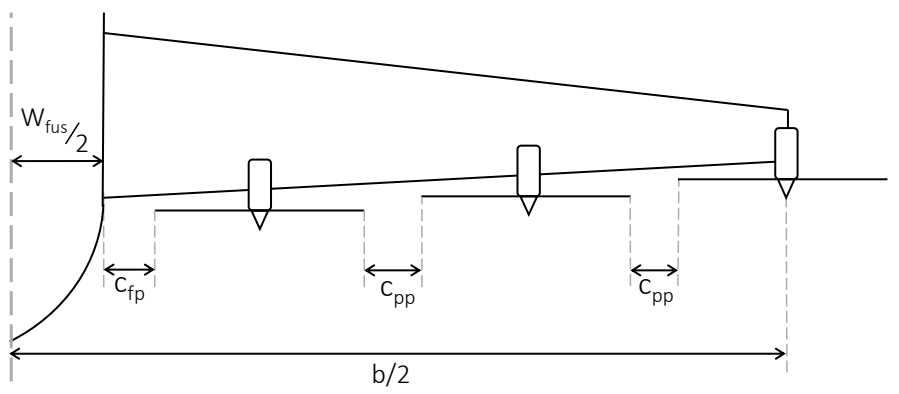

Figure 5. Definition of the fuselage-propeller and propeller-propeller clearances used in the lateral positioning of the propellers.

The leftmost vertical dashed line in Figure 5 is the symmetry plane of the aircraft halfway through the fuselage. The distance to the wingtip from this line is the half-span, $b / 2$. Part of this length is taken by half of the fuselage width, $w_{f u s} / 2$. To prevent problems with wing positioning and rotation, it was decided to define the required fuselage-propeller clearance, $c_{f p}$, with respect to the maximum width of the fuselage, and not the local width. This ensures that, even when the wings are in vertical position, there is sufficient clearance between the inner propellers and the fuselage, without the need for having a detailed design of the fuselage shape at the position of the propellers. The distance between propeller tips is defined by $c_{p p}$, the propeller-propeller clearance. These two clearances are inputs to the method which may be obtained from sizing constraints or estimated based on reference aircraft. The remaining width is taken by the two inboard propellers and half of the tip propeller. This is generalised in Figure 5 for an arbitrary number of propellers, where $N_{\text {prop }}$ is the number of propellers per half wing:

$$
R=\frac{\frac{b}{2}-\frac{w_{f u s}}{2}-c_{f p}-\left(N_{p r o p}-1\right) c_{p p}}{2 N_{p r o p}-1}
$$

\subsection{Analysis of the Blades in Off-Design Conditions}

The resulting geometry is optimised for a chosen design condition, however, due to the nature of eVTOL operations, the propellers encounter a very wide range of operating conditions at different required thrust levels. Thus, the propellers need to be analysed in off-design conditions to verify whether they can achieve enough thrust during all flight phases, including hover. The paper by Adkins and Liebeck, in which the design procedure is presented, also features an analysis procedure for off-design conditions using BEMT [5]. This procedure takes into account the fully defined propeller from which the radius, number of blades, and blade geometry are known, together with the flight conditions and rpm.

The thrust and torque per unit radius can be defined as Equations (7) and (8), respectively [5]:

$$
\begin{gathered}
T^{\prime}=C_{y} \frac{1}{2} \rho w^{2} B c \\
Q^{\prime} / r=C_{x} \frac{1}{2} \rho w^{2} B c
\end{gathered}
$$

These equations make use of the coefficients $C_{y}$ and $C_{x}$, which are related to the lift and drag coefficients of the blade sections, as shown in Equations (9) and (10). A visual representation of the definition of these coefficients on the blade is shown in Figure 4: 


$$
\begin{aligned}
& C_{y}=C_{l} \cos (\Phi)-C_{d} \sin (\Phi) \\
& C_{x}=C_{l} \sin (\Phi)+C_{d} \cos (\Phi)
\end{aligned}
$$

$B$ is the number of blades, $c$ is the chord of the section, $\rho$ is the air density, and $w$ is the local effective flow velocity at the section. The first three parameters are known inputs to the analysis, but the local flow velocity needs to be calculated using Equation (11). The airspeed $V$, rotational speed $\Omega$ and local radius are known inputs, but the axial and radial interference factors, $a$ and $a^{\prime}$, need to be iteratively calculated. This iterative process is also detailed in the original paper [5] —and hence not detailed here:

$$
w=\sqrt{(V(1+a))^{2}+\left(\Omega r\left(1-a^{\prime}\right)\right)^{2}}
$$

\subsection{Noise Analysis}

One key aspect of aircraft that operate in urban environments is their noise emission. Noise can have a negative effect on social sustainability and thus on the acceptance of new urban air mobility solutions. Moreover, noise is regulated, and if the noise emissions of the aircraft are not within regulations, the aircraft cannot fly. In the particular case of the analysed configuration, with open rotors, the majority of the noise comes from the propellers. It is thus paramount to include a preliminary method to estimate the propeller noise in this design framework. The propeller noise under cruise conditions was analysed following a semi-empirical procedure obtained from [14]. This method is for isolated propellers, and hence only serves as a preliminary estimation for multi-rotor configurations in which the noise is affected by the interactions between the propellers (see Section 3.3 for further recommendations).

The formula used to calculate the far-field noise (defined as noise at a distance further than one diameter of the propeller) for a single propeller is shown in Equation (12) [14]:

$$
S P L=L_{1}+20 \log _{10}\left(\frac{4}{B}\right)+40 \log _{10}\left(\frac{15.5}{D}\right)+C_{\text {Mach }}+C_{\theta}-20 \log _{10}(r-1)
$$

In this equation, the first term $L_{1}$ is the reference noise level; it is based on the motor power and it is obtained from Figure B.2 in [14]. The next two terms are corrections for the number of blades, $B$, and propeller diameter, $D$ (in $\mathrm{ft}$ ). The next term, $C_{\text {Mach }}$, is a correction for the tip Mach number, obtained from Figure B-3 in [14]. $C_{\theta}$ is a correction which accounts for the direction in which the noise is being calculated and it is obtained from Figure B-8 in [14]. For this calculation, it was decided to use a correction of $+4 \mathrm{~dB}$, which is the maximum value of the average curve, to obtain the noise at the position in which it is at a maximum. Lastly, the last term, where $r$ is the distance in $\mathrm{ft}$ at which the noise is to be calculated, accounts for the noise attenuation due to propagation from source to observer.

As shown in Equation (12), a higher number of blades results in less noise. This is due to the fact that with more blades, the pressure increment to the flow is introduced in a more distributed manner, which favours selecting a high number of blades for the design of the propellers.

Equation (12) holds for one propeller, so to calculate the combined noise from all propellers, Equation (13) is used, where $L$ represents the noise in $\mathrm{dB}$. This equation results in the sum of the noise by each propeller when calculated under isolated conditions and assuming incoherent sources, but it does not take into account the aerodynamic and aeroacoustic interactions between the propellers. These effects are difficult to model and thus not taken into account in this preliminary analysis; therefore, more accurate aeroacoustic simulations of the chosen architecture are needed and belong to the next steps towards a more detailed design: 


$$
L_{\text {tot }}=10 \log _{10}\left(\sum_{i=1}^{n} 10^{\left(L_{\text {prop }, i} / 10\right)}\right)
$$

\section{Results and Discussion of the Propulsion System Design}

The main design goal is to achieve a propeller that is optimised for cruising, since cruising represents the majority of the mission time for the Wigeon eVTOL investigated herein. This approach does not necessarily result in the propeller design that will minimise the energy consumption over the whole mission but allows one to select a single design-point which will be reasonably close to the optimum without the need for an optimisation for the whole mission profile. However, sizing the propellers with only the cruise requirements results in small propellers that do not allow one to reach the necessary thrust requirements of other mission phases, such as hover. Thus, an extra factor is applied to the target design thrust to obtain a propeller whose thrust range is wide enough to satisfy all mission phases. The procedure to select this factor is to first try a factor of 1 that multiplies the cruise thrust, and then check the maximum available thrust with the resulting propeller geometry for the other mission phases, and accept the design if the design meets the thrust requirements. If not, the factor is increased and the procedure is iteratively repeated. With this, a factor of 2.6 is obtained as the minimum factor to achieve the necessary thrust for all mission stages other than cruising. This factor, applied to the cruise thrust of $153.63 \mathrm{~N}$ per propeller, results in a design thrust of $400 \mathrm{~N}$ per propeller. The flight conditions at the design point are a cruise speed of $72.19 \mathrm{~m} / \mathrm{s}$ at a height of $1 \mathrm{~km}$. The atmospheric values for density, temperature, and dynamic viscosity were obtained from the International Standard Atmosphere.

Before designing the blades, the propeller dimensions need to be defined. For the Wigeon, all clearances are assumed to be $0.3 \mathrm{~m}$. This value was selected to be in the order of magnitude of the expected propeller radius, but it has not been a result of a detailed analysis since the effects of propeller clearance are not accounted for in this design framework; this is further explained in Section 3.3. The effect that these clearances have on the propeller performance and noise are very complex and cannot be calculated with the tools presented in this preliminary design framework, and they remain as future work for further design stages of the aircraft. The half span and fuselage width came from aerodynamic and cabin design requirements detailed in the original project report [3]. Only the number of propellers remains to be chosen in order to obtain the propeller radius. From the shortlisted possibilities of the number of propellers $(8,12$, and 16), having eight propellers was ruled out because maximising the radius would lead to propellers with a $1 \mathrm{~m}$ radius, which would not be acceptable for ground clearance requirements when the wings are in the horizontal position. Having eight propellers with smaller (non-maximised) radius would be a solution to this, but it would eliminate the benefits of having less propellers. Having 16 propellers would lead to propellers with only $0.3 \mathrm{~m}$ of radius and a hover disk loading above $600 \mathrm{~kg} / \mathrm{m}^{2}$. The small radius would already pose problems with the wing and nacelle blocking the slipstream. Moreover, a disk loading of $600 \mathrm{~kg} / \mathrm{m}^{2}$ is above the desired value for the Wigeon, as it is closer to the expected disk loadings of reference lift-fan architectures than to that of tilt-wing aircraft [15]. Therefore, the selected number of propellers is 12 , meaning 3 propellers per half wing.

For 3 propellers per half wing - with clearances of $0.3 \mathrm{~m}$, a fuselage width of $1.38 \mathrm{~m}$ and a span of $8.2 \mathrm{~m}$-the final radius of the propellers is $0.5029 \mathrm{~m}$, resulting in a disk loading of $317.25 \mathrm{~kg} / \mathrm{m}^{2}$. These dimensions also define the spanwise positioning of the propellers, as defined in Figure 5.

The chosen number of blades of the propeller is 6 . This number is based on the noise and manufacturing considerations previously mentioned, and on that fact that more blades result in higher efficiency, as seen in the sensitivity analysis presented in Section 3.2.1. Moreover, the non-dimensional hub radius, $\xi_{0}$, is assumed to be 0.1 . 
The last parameter needed is the rpm at the design condition. The maximum allowable rpm level is 4791 based on a limit tip Mach number of 0.75 . Going beyond this Mach number may result in shock waves at the tips that would lead to additional losses due to wave drag and to high noise levels. The exact maximum allowable Mach number beyond which shock waves develop depends on the airfoil and loading conditions of the blade, and may be in excess of 0.75 , but this number is chosen to simplify the analysis procedure presented herein while ensuring that the maximum value is below a safe threshold. With this maximum value in mind, it was chosen to design the propellers for $1350 \mathrm{rpm}$ at the design condition. This rpm value, below $30 \%$ of the maximum allowable number, is needed to ensure that there is enough rotational speed margin to meet the maximum thrust requirements at maximum rotational speed. The pitch of the blades is also altered to achieve this difference in thrust, but the wide range of thrust conditions cannot solely be achieved by pitch variation. The resulting propeller has an efficiency of $81 \%$ at the design condition and a solidity of 0.36 . The propeller solidity represents how much area of the propeller disk is occupied by the blades. The reference pitch setting at the design condition, defined as the pitch at $70 \%$ of the propeller radius, is 66 degrees.

The next step to analyse the propellers is to see whether they can meet the maximum thrust requirement. For controllability in OEI conditions, the thrust-to-weight ratio needed is 1.5 (this requirement is obtained from a controllability analysis performed in the original project, as can be seen in [3] for reference). With an MTOM of $3025 \mathrm{~kg}$ and 12 propellers, this results in a required maximum thrust of $3708 \mathrm{~N}$ per propeller. This value includes contingencies: the systems of the Wigeon are sized for an operating empty mass (OEM) $10 \%$ higher than the predicted one, to ensure that the design would work in the event that the predicted mass is underestimated, as specified in the original project [3]. In order to achieve this, the propellers are accelerated up to the maximum rpm (4791), and the blades are pitched down by 45 degrees with respect to the design condition; this means reducing $\beta$ in Figure 4. This results in a thrust of $3745 \mathrm{~N}$ per propeller. The efficiency of this condition is $39 \%$, a low value which is expected due to the high disk loading, $317 \mathrm{~kg} / \mathrm{m}^{2}$, and the low advance ratio, $\mathrm{J}=0.12$ (at the assumed $10 \mathrm{~m} / \mathrm{s}$ inflow velocity). These values are obtained at a height of 500 metres, with the corresponding air density, temperature, and dynamic viscosity being obtained from the International Standard Atmosphere. The reason why this height was chosen instead of sea level was to allow for a safety margin and for controllability in higher operations. It is worth noting that the inflow speed for this condition was chosen to be $10 \mathrm{~m} / \mathrm{s}$. This is because the analysis method does not work for inflow speeds of $0 \mathrm{~m} / \mathrm{s}$. However, with lower inflow speed, the thrust of the propeller increases [16] due to the increase in the angle of attack of the blades, which means that this is a conservative estimate. Having this conservative estimate for maximum thrust is also good for the design, since some of the effects are not modelled. The assumption of the thrust increasing under static conditions holds unless the propeller blades suffer significant separation under this condition. This cannot be analysed with the current tools, hence verifying the validity of this assumption for the obtained design remains a point of future work for further stages of the design-for example, by performing a numerical simulation at this condition with the obtained geometry from the design framework.

The next step was to analyse the propellers in stable hover. With an MTOM of $3024.8 \mathrm{~kg}$ (using contingencies) and 12 propellers, the thrust per propeller required to hover is $2472 \mathrm{~N}$. By accelerating the propeller to $4000 \mathrm{rpm}$ and reducing the pitch angle of the blades by 45 degrees, the necessary thrust was achieved. This was also calculated at a height of 500 metres above sea level. The efficiency at this condition was $41 \%$, and the advance ratio was 0.15 .

The last step is the nominal cruise condition analysis as the design point for a higher thrust level. To achieve the cruise thrust of $154 \mathrm{~N}$, the pitch was kept at the design condition and the rpm of the propeller was reduced to $1090 \mathrm{rpm}$, resulting in a cruise thrust per propeller of $158 \mathrm{~N}$. At this condition, the efficiency was $77 \%$, four points lower than under the design condition and the advance ratio was 3.2. 
The results from these analyses can be found in Table 1 and the resulting propeller geometry can be seen in Figures 6-8.

Table 1. Thrust settings at cruise, hover, and full thrust conditions (values per propeller).

\begin{tabular}{llll}
\hline Parameter & Cruise & Hover & Full Thrust \\
\hline Thrust (N) & 158 & 2502.42 & 3745.14 \\
RPM & 1090 & 4000 & 4791 \\
$\Delta \beta$ (deg) & 0 & -44 & -45 \\
\hline & & & \\
& & &
\end{tabular}

Figure 6. Side view of the blade angle under cruise conditions.

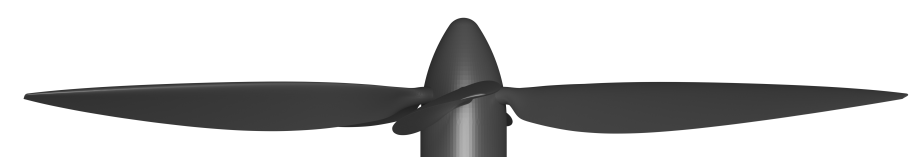

Figure 7. Side view of the blade angle under hovering conditions.

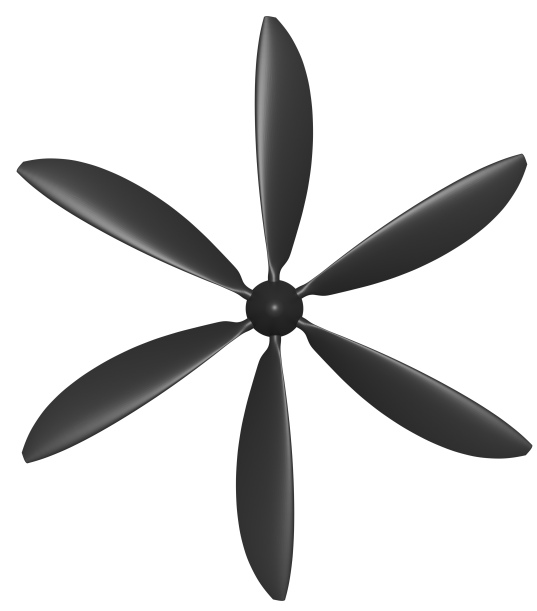

Figure 8. Top view of the optimised propeller geometry under hovering conditions.

The positioning of the propellers is shown in Figure 9. As can be seen, the overlap between the front and back propellers was minimised with the chosen number of propellers and their size to prevent the noise and performance penalties that slipstream ingestion brings. Note that the position of the wings stems not only from this consideration, but also from further aerodynamic considerations detailed in the original project [3].

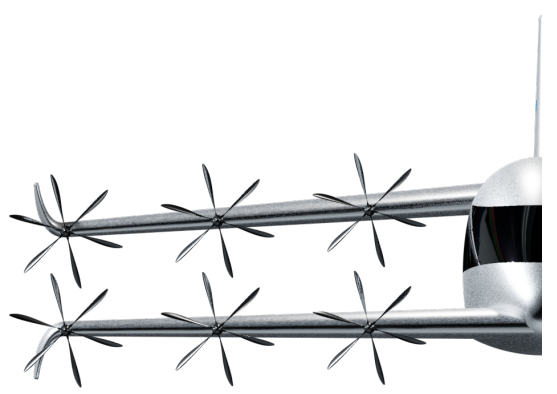

Figure 9. Front view of the aircraft showing the propeller positioning. 


\subsection{Noise Analysis Results}

Under cruise conditions, the reference noise level $L_{1}$ for the Wigeon was determined to be $107 \mathrm{~dB}$. The tip Mach number for the Wigeon is slightly below 0.3 , for which $C_{\text {Mach }}$ is $-19 \mathrm{~dB}$. Using Equation (12) with these values, the noise from one propeller during cruise at $100 \mathrm{~m}$ from the aircraft was determined to be $65.06 \mathrm{~dB}$. The $100 \mathrm{~m}$ distance was chosen as a sample distance to evaluate the noise. Similarly, for $1000 \mathrm{~m}$, the noise representing the cruise height is $45.03 \mathrm{~dB}$. For the 12 propellers combined, the noise levels at $100 \mathrm{~m}$ and $1000 \mathrm{~m}$ are $75.85 \mathrm{~dB}$ and $55.83 \mathrm{~dB}$, respectively. The semi-empirical method used was not developed for highly loaded propellers under hovering conditions, and as such, only cruise estimates were presented here. Since hovering is a critical part of the noise performance of eVTOLs, this remains an important point for future work, as mentioned in Section 3.3.

\subsection{Sensitivity Analysis}

In order to see the effects of the input parameters on the results of the propeller design and analysis, a sensitivity analysis was performed.

\subsubsection{Sensitivity of the Design Procedure}

The first part of the sensitivity analysis concerns the blade design procedure explained in Section 2.1. For this procedure, the sensitivity analysis was divided into two parts: one concerning the inputs that are not a design choice (freestream velocity which comes from the flight performance analysis performed in the original project [3], and the propeller radius, which is bounded by the procedure laid down in Section 2.2); and one concerning the design variables that can be freely chosen while designing the propeller (propeller rpm and number of blades). Since the goal of the procedure is to maximise efficiency at a given thrust setting, the parameter of interest is the efficiency of the propeller. The results of this sensitivity analysis can be seen in Figures 10 and 11.

From Figure 10, it can be seen that a higher radius increases efficiency, which is expected as it decreases disk loading. This leads to the design goal of maximising the radius of the propellers. The design point for the cruise speed $V$, since it is selected based on requirements of the mission design in the original project [3], and is affected by other design disciplines, could not be chosen during the design process of the propellers, but it is still important to analyse the effect that it has on propeller performance. The graph includes the final design point for cruising conditions.

From Figure 11, it can be seen that more blades mean more efficiency, which partly explains the reasoning behind the selected number of blades, as explained in Section 3. The increase in efficiency is due to an increase in the induced efficiency of the blades [6]. However, with the method shown in Section 2.1, the total solidity of the propeller remains nearly constant independently of the selected number of blades, which results in blades with a lower chord length for a higher number of blades, which results in a decrease in profile efficiency due to the lower Reynolds number of the blade [6]. Overall, the effect of the number of blades on the final result is not very high, especially close to the design point. For the rotational speed, the design value chosen was below the optimal one. The reason for this is that the thrust needed for cruising is very low compared to the maximum thrust required, and this last value needs to be achieved at a rotational speed of at most $4790 \mathrm{rpm}$, and thus this margin is needed. 


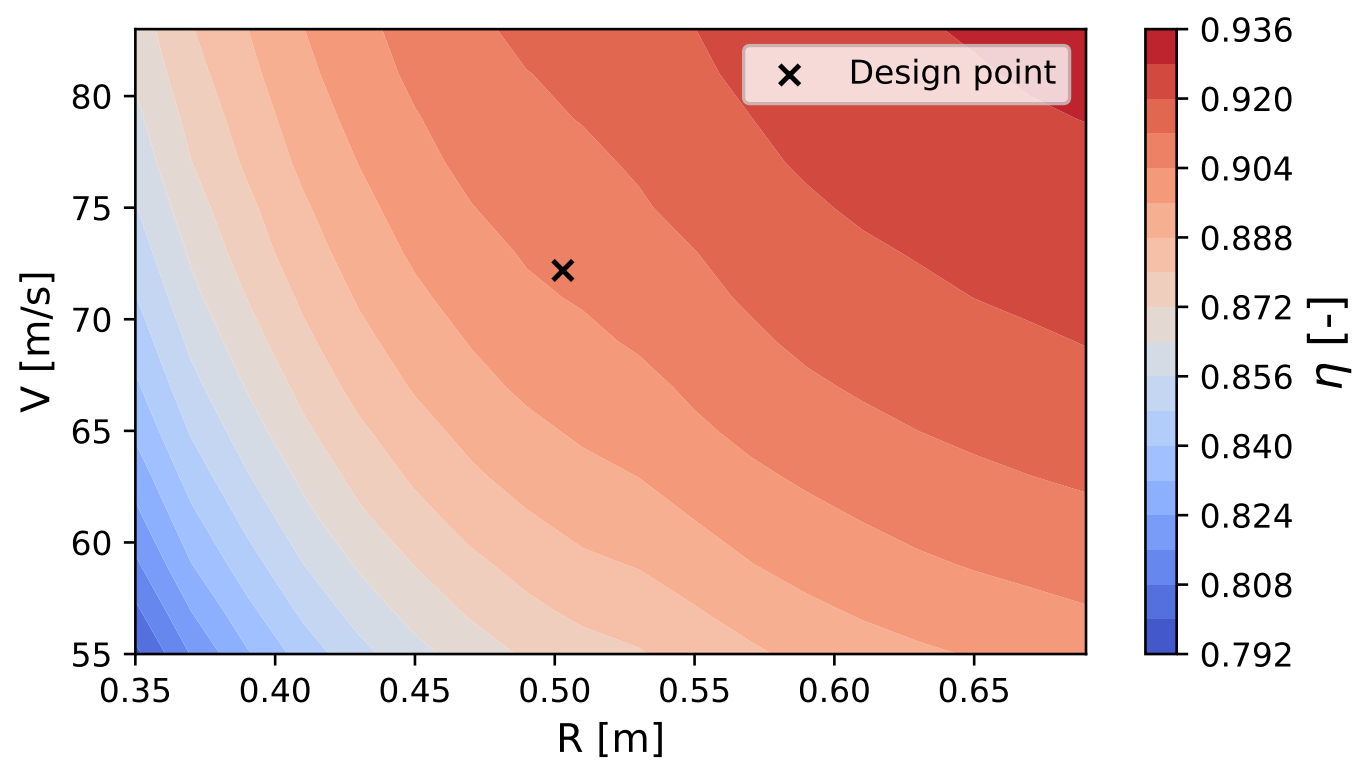

Figure 10. Sensitivity analysis of propeller efficiency in the design procedure to variations in freestream velocity and propeller radius.

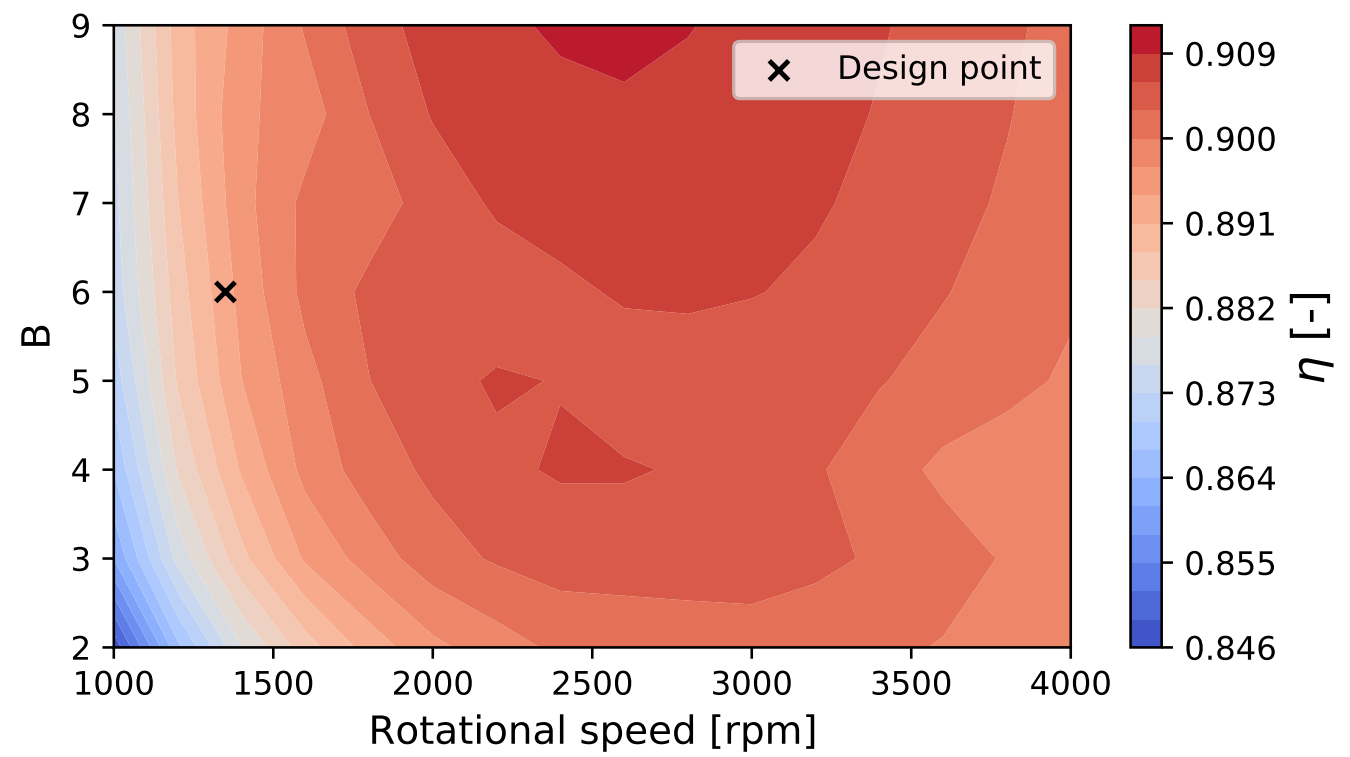

Figure 11. Sensitivity analysis of propeller efficiency in the design procedure to variations in rpm and the number of blades.

\subsubsection{Sensitivity of the Propeller Analysis}

The second part of the analysis concerns the procedure to analyse the propeller under off-design conditions. For this part, the sensitivity analysis was conducted for both the effect on propeller efficiency, shown in Figure 12, and on thrust, shown in Figure 13. These sensitivity analyses show the change in thrust and efficiency with a change in rpm and blade pitch. There is no design point included because this analysis is used to evaluate the performance under arbitrary conditions, not under the design ones. It can be seen that with an increase in rpm, the thrust increases, and thus the efficiency also decreases due to the higher disk loading, as expected. For changes in pitch, the results are no longer linear, because a change in pitch results in a change in the angle of attack of the blade, and thus the lift and drag coefficients, which in turn have an effect on the induction factors $a$ and $a^{\prime}$. These factors affect the velocity seen by the blade, which thus affects the flow angle $\Phi$, and as a result, the aforementioned parameters are affected again. As a result, in order to obtain the best performance when selecting a change in pitch and rpm to meet the thrust 
requirements in the hover and maximum thrust conditions (Section 3), the performance of the propeller is computed over a wide range of pitch angles, after which the best is selected.

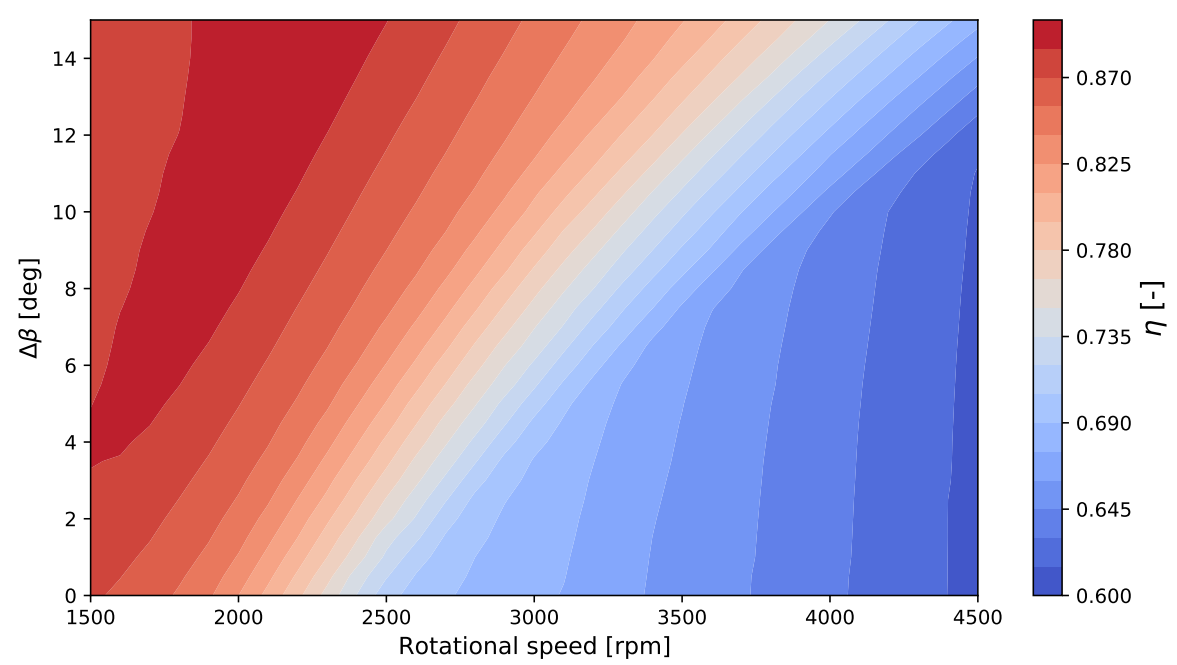

Figure 12. Sensitivity of the efficiency to changes in rpm and pitch angle of the blade in the off-design analysis procedure.

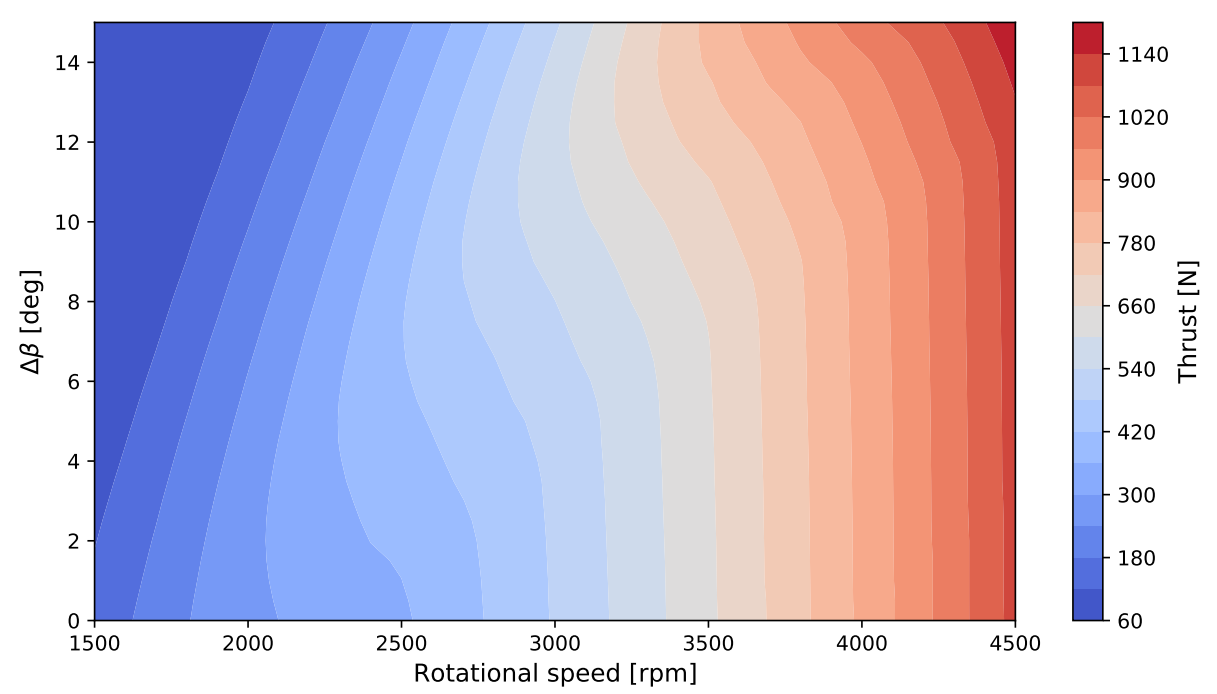

Figure 13. Sensitivity of the thrust to changes in rpm and pitch angle of the blade in the off-design analysis procedure.

\subsection{Future Recommendations on the Propulsion Design}

The proposed design methodology was intended as a preliminary design tool, and as such, several assumptions were made. While some of them were necessary to keep the method simple and sufficiently fast, there are points on the methodology which warrant further research.

Stall of blade sections: The aerodynamic data for the airfoil was computed in XFOIL, which loses accuracy in the post stall region of the lift polar. Since parts of the blades might be stalled during operation due to the wide range of operating conditions, it is important to ensure that the post-stall aerodynamic data of the airfoil is available and accurate. This is one of the points where the procedure needs improvement, and next steps could for example include the CFD simulations of the airfoil to obtain more accurate post-stall data.

Blade design method: The method used to design the blade geometry is not robust when the propellers are too highly loaded, where the iterative blade design procedure given in Section 2.1 can fail to converge. The momentum theory approximation that assumes that 
the increase in flow velocity with respect to the freestream velocity at the disk plane is half the total increase at the wake is not always accurate and can result in convergence issues [5]. Propellers with the moderate-to-high disk loading adopted for the Wigeon $\left(317 \mathrm{~kg} / \mathrm{m}^{2}\right)$ can still be designed with this method, but a different approach would be needed if the chosen architecture features very highly loaded propellers or fans.

Effects of interactions: Due to the complexity of their analysis, the effects of propellerwing and propeller-propeller interaction were not modelled in the present study. Since these interactions can have an effect on propeller performance, quantifying them is important for a more accurate design. For the Wigeon, a propeller lateral separation of $0.3 \mathrm{~m}$ was chosen, which corresponds to $30 \%$ of the radius. A study by de Vries et al. shows that, in horizontal flight, a distributed propulsion system using open rotors sees a drop in propeller efficiency of $1.5 \%$ for a separation between propeller tips of $2 \%$ of the propeller diameter [8]. In a different study carried out by Zhou et al., it was shown that the thrust coefficient of two propellers with a tip separation of $5 \%$ of the propeller diameter is within $2 \%$ of that of a single propeller under static thrust conditions, although a significant increase in thrust fluctuation is seen [9]. Zhou et al. also showed that an increase in noise was observed with decreasing separation [9]. Based on the results from these studies, it is expected that the interaction effects will not cause significant deviation from the predicted performance with the chosen clearance; however, the sensitivity analysis on the effect of this clearance could not be performed with the available tools. In transition, these effects can be more significant [7]. Another effect of propeller-propeller interaction is slipstream ingestion in the second row of propellers, located in the aft wing, and not considered in the present study. Quantifying this interaction is important because it can lead to more significant losses in the calculated thrust [7], while yet negatively affecting noise. Finally, the effect of the propeller blockage due to the wing was not modelled, which can also affect propeller performance, while also affecting the positioning of the propellers with respect to the wing in terms of height and depth.

Airfoil selection: For the present study, a NACA4412 airfoil was assumed, but improved airfoil selection and optimisation were expected in further stages of the design. An early airfoil selection that is more tailored to the configuration and mission of the aircraft to be analysed could already be considered in the preliminary design.

Aeroacoustic simulation: The noise analysis presented herein was based on a semiempirical method which might not be accurate for the Wigeon configuration, especially considering the propeller-wing, propeller-propeller, and wing-wing interactions. Hence, the next design phases should move towards more accurate aeroacoustic simulations of the aircraft based on the geometry obtained from the preliminary design.

The verification of the implementation of the numerical methods was included in [3], in which the outputs from the blade design method were compared against actuator disk theory and against expected trends for the efficiency with a varying advance ratio. The output from the presented design framework allows one to build relatively accurate CAD models of the propellers which can then be used in numerical simulations to analyse the more complex phenomena which have not been included in the preliminary design and which can be used to assess the performance of specific designs obtained from the presented method.

\section{Power System Design Methodology}

The goals of the preliminary power system design are to obtain a mass estimate for the power system and to develop an initial configuration for the batteries.

The method described herein is based on the required maximum power and required total energy. The inputs used to obtain the results mentioned in Section 5 come from [3], which is the original project for which the present methodology was developed. The following inputs are taken into account: the required energy stored, the required maximum power provided, the properties of individual battery cells, the division of battery cells for the motors and other systems, and redundancy. 


\subsection{Mass and Weight Calculations}

The following formulae give the mass of the battery package, based on the required energy (Equation (14)) and maximum power (Equation (15)); where DoD stands for depth of discharge and EOLC stands for end-of-life capacity, both having a value between 0 and 1:

$$
\begin{aligned}
m_{\text {bat }} & =\frac{E_{\mathrm{req}}}{E_{\mathrm{sp}}} \frac{1}{\mathrm{DoD} \times \mathrm{EOLC}} \\
m_{\text {bat }} & =\frac{P_{\max }}{P_{\mathrm{den}}} \frac{1}{\mathrm{DoD} \times \mathrm{EOLC}}
\end{aligned}
$$

The mass of the final battery was the larger of the two values calculated with Equations (14) and (15). The volume of the battery can then be determined with Equation (16):

$$
v_{b a t}=m_{b a t} \times \frac{E_{\mathrm{sp}}}{E_{\mathrm{vol}}}
$$

where $E_{\mathrm{sp}}$ is the specific energy in $\mathrm{Wh} / \mathrm{kg}$ and $E_{\mathrm{vol}}$ is the volumetric energy density in $\mathrm{Wh} / \mathrm{m}^{3}$.

After determining the mass and volume of the battery pack, the next step is to obtain the masses for the other parts of the powertrain. The two parts that are considered in the preliminary design are the motor controllers and the electric motors. This is done by multiplying the maximum power by the combined specific power of the motor controllers and the electric motors. This combined specific power is calculated with Equation (17):

$$
P_{\mathrm{sp}, \mathrm{tot}}=\left(\frac{1}{P_{\mathrm{sp}, \mathrm{mot}}}+\frac{1}{P_{\mathrm{sp}, \mathrm{mc}}}\right)^{-1}
$$

\subsection{Battery Configuration}

Not only is the size of the battery important for the design, but so are the specifications in terms of configuration. A battery consists of a number of battery cells. The number of cells depends on the properties of the individual cells and the required properties of the total battery.

Some parameters other than total battery size play a role in the preliminary design of the batteries, of which safety and ease of work are used in the preliminary design phase. A method that is considered to increase the safety consists of adding two motor controllers per motor, instead of one, allowing a redundant construction. However, duplicated motor controllers would result in a significant weight penalty given that each controller weighs approximately $11 \mathrm{~kg}$ based on the power density of [17], thus resulting in a total weight increase of approximately $130 \mathrm{~kg}$. In addition to this, the Wigeon eVTOL was designed to withstand a single motor failure, and thus the weight penalty was viewed as too high to accept additional redundancies. The original analysis for the OEI condition can be found in [3].

Therefore, the main strategy adopted to achieve the safety of the power system for the present design consists of limiting the impact of battery failure. By having two batteries per electric motor, such redundancy can be achieved, such that in the case of a battery failure, the affected motor still has half of the original energy at its disposal. By assuming that all the battery packs are equal, a modular design can be achieved, making the aircraft easier to manufacture and maintain. The aircraft has an additional, dedicated battery for the non-propulsive parts of the power system, but this battery is not the focus of the preliminary design methodology presented herein.

There are two ways of connecting batteries: in series or in parallel. Batteries connected in series have the same current, and the total voltage of batteries in series is the sum of their individual voltages, represented by Equation (18). Batteries connected in parallel, on the other hand, experience the same voltage and the total current is the sum of their individual currents. 
Equation (19) gives the total energy $E$ stored in a single battery cell. This is found by multiplying the capacity $C$ and the nominal cell voltage $V$. The values for $C$ and $V$ have a major influence on the relative increase in the number of cells. This effect is described in more detail in Section 5.4. The values for $C$ and $V$ were chosen to minimise this increase:

$$
\begin{aligned}
V_{\text {tot }} & =\sum_{i=1}^{n} V_{i} \\
E & =C V
\end{aligned}
$$

Equation (20) was used to calculate the number of cells required for the electric motors only based on the required energy for the propulsion system. Equation (21) is used to calculate the number of cells for the remaining batteries. These values have to be rounded up to the next integer:

$$
\begin{gathered}
n_{c_{\text {mot }}}=\left\lceil\frac{E_{\text {tot }} \times \%_{\text {mot }}}{100 E_{c}}\right\rceil \\
n_{c_{\text {misc }}}=\left\lceil\frac{E_{t o t}\left(100-\%_{m o t}\right)}{100 E_{c}}\right\rceil
\end{gathered}
$$

where $n_{c}$ is the number of cells, $\%_{m o t}$ is the percentage of energy that goes into the motors and $E_{c}$ is the energy stored in a single cell. These two are separated as a consequence of the battery configuration, since both the propulsive and non-propulsive power storages need to have an integer number of cells.

The initial number of cells from Equation (20) does not take into account the fact that a battery not only needs to provide energy, but also the correct voltage. The number of cells in series is determined by the required voltage for the electric motors, and can be found by rewriting Equation (18) into Equation (22). Dividing the total number of cells from Equation (20) by the number of cells in series gives the number of cells in parallel, as stated in Equation (23):

$$
\begin{aligned}
& n_{\mathcal{c}_{s e r}}=\left\lceil\frac{V_{m o t}}{V_{c}}\right\rceil \\
& n_{c_{\text {par }}}=\left\lceil\frac{n_{\mathcal{c}_{\text {mot }}}}{n_{\mathcal{c}_{\text {ser }}}}\right\rceil
\end{aligned}
$$

Since both the number of cells in parallel and in series are increased due to rounding, the total number of cells has increased. The new total number of battery cells of the power system can then be calculated using Equation (24). The percentage increase in battery cells with respect to the original number from Equation (20) can be calculated using Equation (25):

$$
\begin{gathered}
n_{c_{\text {new }}}=n_{\text {cpar }_{\text {par }}} \times n_{\mathcal{c}_{\text {ser }}} \\
\Delta n_{c}=\frac{n_{c_{\text {new }}}-n_{\mathcal{c}_{\text {old }}}}{n_{c_{\text {old }}}} \times 100 \%
\end{gathered}
$$

Until this point, all calculations have been solely dependent on the total required energy. From here onwards, however, the required number of modules for the battery will take a leading role. However, the power system is being designed for a tandemwing eVTOL aircraft with distributed propulsion and two batteries per motor to increase reliability. To ensure modularity, which is convenient for manufacturing and maintenance, it is required that all batteries have the same number of cells in parallel. This means that the number of cells in parallel has to be scaled up to the nearest number that is a multiple of the total number of individual batteries. This way, each battery has the same number of cells in parallel, therefore, they all have the same construction. This allows the batteries to be easily replaced, as all battery packs are identical. 


\section{Results and Discussion of the Power System Design \\ 5.1. Battery Characteristics}

The battery type chosen is a solid-state lithium battery, and more details on the battery selection can be found in [2]. Since the Wigeon is designed to be released in 2030, futureoriented battery specifications can be used. The assumed batteries have a specific energy of $500 \mathrm{Wh} / \mathrm{kg}$, a volumetric energy density of $1000 \mathrm{Wh} / \mathrm{L}$, and a power density of $6500 \mathrm{~W} / \mathrm{kg}$. These values were based on [18] and were adapted herein to fit a commercial product.

The depth of discharge (DoD) chosen was $80 \%$ or 0.8 . There are cells in development, designed to perform at close to $100 \%$ DoD whilst also having an operational life of over 800 cycles [19]. For further calculations, a DoD of $80 \%$ was chosen as a precaution, since the batteries mentioned in [19] are still in the experimental phase, and the final DoD could turn out to be less. This $80 \%$ DoD means that the battery has a deep discharge, as the DoD is higher than 50\%. From [18], it follows that the battery can withstand these discharges over the design life. At the end of life, $85 \%$ of the original capacity should remain. Based on three flights a day, this end-of-life capacity (EOLC) will be reached after 7.5 years. Since the operational lifetime for the Wigeon eVTOL is estimated to be 15 years [3], this means that the batteries will only require one change over its lifetime. The final characteristic of the batteries is that they charge quickly, with an $80 \%$ state of charge reached in 15 min [19] and the estimated time for a full charge being $25 \mathrm{~min}$.

Each individual battery cell has a nominal voltage of $3.7 \mathrm{~V}$ and an internal capacitance of $5 \mathrm{Ah}$, based on current battery cells [20].

\subsection{Powertrain Sizing}

The required energy for a full flight of the Wigeon is $301 \mathrm{kWh}$, and the maximum power is 1.8 MW [3]. Using these values and Equations (14) and (15), it was found that the battery mass required for energy storage is $886 \mathrm{~kg}$, and that the battery mass required for maximum power is $409 \mathrm{~kg}$. Thus, for the Wigeon the batteries design is energy driven and not power driven, achieving a total battery mass of $886 \mathrm{~kg}$. With Equation (16), the volume is computed at $0.443 \mathrm{~m}^{3}$.

Based on values from Calnetix [21] and magniX [17], a combined specific power for the motor controllers and the electric motors was found using Equation (17), this being $6800 \mathrm{~W} / \mathrm{kg}$. To prevent an overestimation of the power density, which would have a major effect on the MTOM, the calculations were performed with a lower power density: $5000 \mathrm{~W} / \mathrm{kg}$. Using this power density resulted in a total mass for the combined motor controllers and electric motors of $503 \mathrm{~kg}$.

\subsection{Battery Configuration}

Using the nominal voltage and capacitance from Section 5.1 and Equation (19), the total energy per cell was computed to be $18.5 \mathrm{Wh}$. An analysis of the vehicle's power budget showed that the power required for components other than the propulsion system amounts to approximately $1 \%$ of the total power required [3] (Section 9.3). Using this information and Equations (20) and (21), it was found that there are 16,114 cells required for the energy storage for the propulsion system, and 163 cells for other electronic devices, resulting in a total of 16,277 cells. These numbers will increase, since they do not take into account the distribution of cells in series and in parallel.

The next steps solely focus on the cells for the propulsion system. The required voltage for the motors is $500 \mathrm{~V}$, based on Calnetix [21]. The number of cells in series is based on the required voltage, see Equation (22), and is 136. From Equation (23), it follows that the number of cells in parallel is 119 . Due to rounding, this means that the number of battery cells is increased from 16,114 to 16,184 .

Since the Wigeon features 12 propellers (Section 3) and 2 batteries are required per propeller to ensure the aforementioned redundancy, a total of 24 batteries were used. This means that the total number of cells in parallel needs to be a multiple of 24 , to ensure that a modular battery design is possible. The number of battery cells in parallel of the whole 
system increased from 119 to 120 to allow for this. This gives 24 batteries, all with 5 cells in parallel.

The total increase in the number of cells can now be calculated using Equation (25). The number of cells increased by 206 , which is an increase of $1.27 \%$ over the initial value. There are 16,320 cells required for the propulsive system, and 16,483 in total.

\subsection{Sensitivity Analysis of the Power Design Methodology}

Figure 14 displays the relation between the capacity of a single cell and the increase in the number of cells due to the division in cells in series and in parallel, with respect to the number of cells purely required for energy storage for that capacity. The saw-like pattern comes from the fact that the number of total cells in parallel has to be a multiple of the number of batteries. When the capacity increases, the energy stored in each cell, and thus the total energy stored, increases. At certain values for the cell capacity, the total number of cells in parallel can be reduced, while still meeting the minimal energy requirements. This can be seen in Figure 14 as a sudden jump downwards. With an increase in capacity comes an increase in amplitude. The total number of cells decreases when the cell capacity increases, and thus, the percentage of increase in the cell count increases.

Figure 15 shows the relation between the percentage of cells increase and the nominal cell voltage. It can be seen that a small change in nominal voltage can have a major effect on the number of cells. For example, using a nominal cell voltage of $3.71 \mathrm{~V}$ instead of $3.70 \mathrm{~V}$ turns the change in the number of cells from $1.27 \%$ into $20.75 \%$. This is caused by the implementation of the required voltage from a battery pack. This is now exactly $500 \mathrm{~V}$, and the number of cells is chosen to fit this number. Especially when working with low nominal voltages, many spikes are observed, which is not entirely representative of a real-world situation, where: small production differences result in different cell voltages and capacities; and the motors do not exactly require $500 \mathrm{~V}$, but instead have a window of design voltages for which they work as intended. The method can be improved by accounting for ranges for cell voltage, capacity, and the required voltage for the motors. This reduces the peaks in the sawtooth-like shape shown in Figure 15.

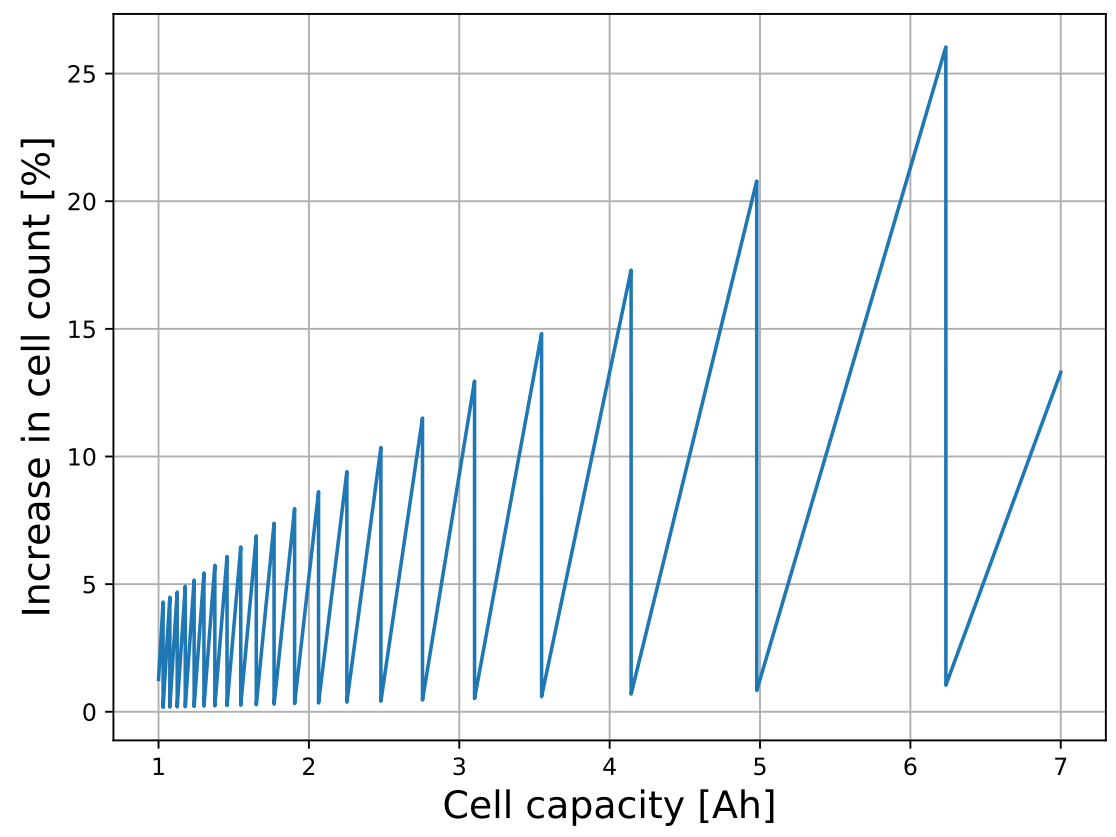

Figure 14. Relation between cell capacity and the increase in the number of cells with respect to the number before taking the configuration into account. 


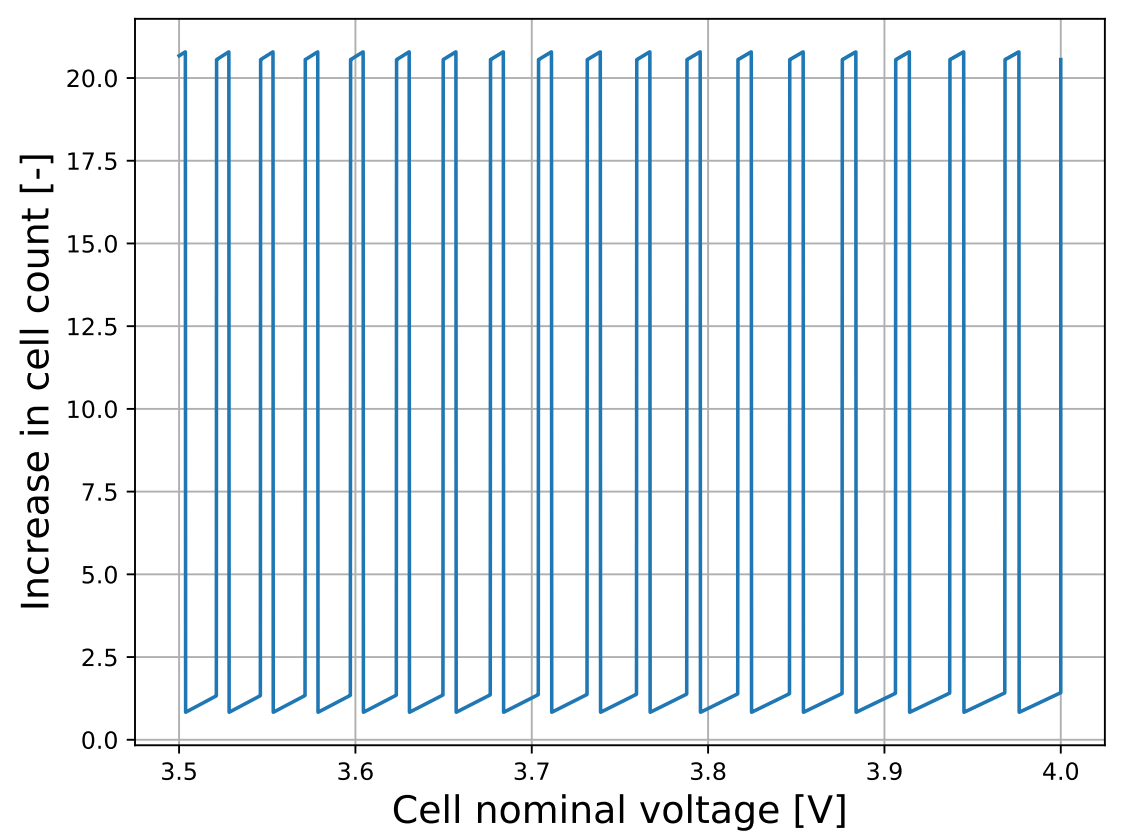

Figure 15. Relation between cell voltage and the increase in the number of cells with respect to the number before taking the configuration into account.

\subsection{Future Recommendations}

The method for determining the battery configuration started from the cell properties and determined the overall battery configuration from there. Alternatively, one could try to start with a battery configuration, and design the optimal battery cell to work with this.

Two further areas where improvements in the method can be made are in the way the configuration is very dependent on the values of a single cell, as clearly shown in Section 5.4. Two things are not taken into account in the method presented for the battery configuration which have a major effect on the sensitivity analysis: both the battery cells and the required voltage for the motors are not fixed values. Instead, both will fall within a range of allowed values that, if considered, would prevent the observed saw-like pattern in the percent increase in cell count.

For validation efforts that should occur later in the detailed design process, a real power system should be constructed and tested, with the first tests performed at a small scale, progressively scaling up towards an individual battery module, until achieving the test of the entire system.

Author Contributions: Conceptualisation, J.A.-M. and K.P.v.R.; data curation, S.G.P.C.; formal analysis, J.A.-M. and K.P.v.R.; investigation, J.A.-M. and K.P.v.R.; methodology, J.A.-M. and K.P.v.R.; project administration, S.G.P.C.; resources, S.G.P.C.; software, J.A.-M. and K.P.v.R.; supervision, T.S. and S.G.P.C.; validation, J.A.-M. and K.P.v.R.; visualisation, J.A.-M. and K.P.v.R.; writing—original draft, J.A.-M. and K.P.v.R.; writing-review and editing, T.S. and S.G.P.C. All authors have read and agreed to the published version of the manuscript.

Funding: This research received no external funding.

Institutional Review Board Statement: Not applicable.

Informed Consent Statement: Not applicable.

Data Availability Statement: Not applicable. 
Acknowledgments: The authors would like to thank the rest of the authors of the original Wigeon project, without whom this work would not have been possible: E. Beyne; M. Buszek; M. CuadratGrzybowski; A. Montoya Santamaría; N. Poliakov; N. Salvador López; J. Schoser; and K. Wadia. We would also like to thank the coaches of the project, D. Biagini and A. Nokhbatolfoghahai. We also thank D. Casalino; J. Dong; G. Eitelberg; Y. Fuerkaiti; R. Nederlof; and D. Ragni for their help and support during the Wigeon project.

Conflicts of Interest: The authors declare no conflict of interest.

\section{Abbreviations}

The following abbreviations are used in this manuscript:

$\begin{array}{ll}\text { ADT } & \text { Actuator Disk Theory } \\ \text { BEMT } & \text { Blade Element Momentum Theory } \\ \text { CAD } & \text { Computer Aided Design } \\ \text { CICD } & \text { Continuous Integration/Continuous Delivery } \\ \text { DEP } & \text { Distributed Electric Propulsion } \\ \text { DOD } & \text { Depth of Discharge } \\ \text { EOLC } & \text { End-of-Life Capacity } \\ \text { eVTOL } & \text { Electric Vertical Take-Off and Landing } \\ \text { MTOM } & \text { Maximum Take-Off Mass } \\ \text { OEI } & \text { One Engine Inoperative } \\ \text { OEM } & \text { Operating Empty Mass } \\ \text { UAM } & \text { Urban Air Mobility } \\ \text { UAV } & \text { Unmanned Aerial Vehicle }\end{array}$

\section{References}

1. Alba-Maestre, J.; Beyne, E.; Buszek, M.; Cuadrat-Grzybowski, M.; Montoya Santamaria, A.; Poliakov, N.; Prud'homme van Reine, K.; Salvador Lopez, N.; Schoser, J.; Wadia, K. Baseline Report-Multi-Disciplinary Design and Optimisation of Long-Range eVTOL Aircraft; Technical Report; Delft University of Technology: Delft, The Netherlands, 2021.

2. Alba-Maestre, J.; Beyne, E.; Buszek, M.; Cuadrat-Grzybowski, M.; Montoya Santamaria, A.; Poliakov, N.; Prud'homme van Reine, K.; Salvador Lopez, N.; Schoser, J.; Wadia, K. Midterm Report-Multi-Disciplinary Design and Optimisation of a Long-Range eVTOL Aircraft; Technical Report; Delft University of Technology: Delft, The Netherlands, 2021.

3. Alba-Maestre, J.; Beyne, E.; Buszek, M.; Cuadrat-Grzybowski, M.; Montoya Santamaria, A.; Poliakov, N.; Prud'homme van Reine, K.; Salvador Lopez, N.; Schoser, J.; Wadia, K. Final Report-Multi-Disciplinary Design and Optimisation of a Long-Range eVTOL Aircraft; Technical Report; Delft University of Technology: Delft, The Netherlands, 2021.

4. EASA. Special Condition for Small-Category VTOL Aircraft. Available online: https://www.easa.europa.eu/sites/default/files/ dfu/SC-VTOL-01.pdf (accessed on 8 November 2021).

5. Adkins, C.; Liebeck, R. Design of optimum propellers. J. Propuls. Power 1994, 10, 676-682. [CrossRef]

6. Larrabee, E.E. Practical Design of Minimum Induced Loss Propellers. SAE Trans. 1979, 88, $2053-2062$.

7. Stokkermans, T.; Usai, D.; Sinnige, T.; Veldhuis, L. Aerodynamic Interaction Effects Between Propellers in Typical eVTOL Vehicle Configurations. J. Aircr. 2021, 58, 815-833. [CrossRef]

8. de Vries, R.; van Arnhem, N.; Sinnige, T.; Vos, R.; Veldhuis, L. Aerodynamic interaction between propellers of a distributedpropulsion system in forward flight. Aerosp. Sci. Technol. 2021, 118, 107009. [CrossRef]

9. Zhou, W.; Ning, Z.; Li, H.; Hu, H. An Experimental Investigation on Rotor-to-Rotor Interactions of Small UAV Propellers. In Proceedings of the 35th AIAA Applied Aerodynamics Conference, Denver, CO, USA, 5-9 June 2017.

10. Betz, A. Screw Propellers with Minimum Energy Loss; Technical Translation; NRC, Division of Mechanical Engineering: Ottawa, ON, Canada, 1958.

11. XFOIL—Subsonic Airfoil Development System. Available online: https://web.mit.edu/drela/Public/web/xfoil/ (accessed on 19 October 2021).

12. Nederlof, R. Improved Modeling of Propeller-Wing Interactions with a Lifting-Line Approach; Technical Report; Delft University of Technology: Delft, The Netherlands 2020.

13. Sinnige, T.; van Arnhem, N.; Stokkermans, T.; Eitelberg, G.; Veldhuis, L. Wingtip-Mounted Propellers: Aerodynamic Analysis of Interaction Effects and Comparison with Conventional Layout. J. Aircr. 2019, 56, 295-312. [CrossRef]

14. Marte, J.E.; Kurtz, D.W. A Review of Aerodynamic Noise From Propellers, Rotors, and Lift Fans; Technical Report; NASA: Pasadena, CA, USA 1970.

15. Filipenko, M. Back to the Future: A Rough Comparison of 70s VTOL Concepts to Current eVTOL Designs. Available online: https: //www.linkedin.com/pulse/back-future-rough-comparison-70s-vtol-concepts-evtol-filipenko/ (accessed on 20 October 2021). 
16. MH Aerotools. Static Thrust of Propellers. Available online: https://www.mh-aerotools.de/airfoils/prpstati.htm (accessed on 21 June 2021).

17. magniX-magniDrive 100. Available online: https:/ / www.magnix.aero/products (accessed on 29 June 2021).

18. Luhan, Y.; Xin, L.; Paulson, J.A. A dynamic stability design strategy for lithium metal solid state batteries. Nature 2021, $593,218-222$.

19. Next-Generation Solid-State Batteries. Available online: https://www.quantumscape.com/wp-content/uploads/2021/02/DataLaunch-Updated-Post-Presentation-20210107-2.pdf (accessed on 29 June 2021).

20. Lithium Polymer (Li-Po) Batteries/Cells. Available online: https://melasta.com/cells/lithium-polymer-li-po-batteries-cells.html (accessed on 29 June 2021).

21. High Speed Electric Motor Generators. Available online: https://www.calnetix.com/high-speed-electric-motor-generators (accessed on 29 June 2021). 